\title{
MACULOPATÍA POR TAMOXIFENO. ESTUDIO MEDIANTE LA TOMOGRAFÍA DE COHERENCIA ÓPTICA
}

\section{OPTICAL COHERENCE TOMOGRAPHY STUDY IN TAMOXIFEN MACULOPATHY}

\author{
BAGET-BERNALDIZ $\mathrm{M}^{1}$, SOLER LLUIS $\mathrm{N}^{1}$, ROMERO-AROCA ${ }^{2}$, TRAVESET-MAESO A ${ }^{1}$
}

\section{RESUMEN}

Caso clínico: Se describe el caso de una paciente que acudió por pérdida bilateral y progresiva de la visión después de haber recibido tratamiento con tamoxifeno durante 13 años. Para el estudio detallado de las alteraciones maculares se realizaron la angiografía fluoresceínica y la tomografía de coherencia óptica.

Discusión: La pérdida de visión debido a la maculopatía por tamoxifeno puede ser debida tanto a la atrofia de las capas de fibras nerviosas de la retina como a la presencia de edema macular. Los hallazgos maculares obtenidos mediante la angiografía fluoresceínica y la tomografía de coherencia óptica son complementarios.

Palabras clave: Maculopatía por tamoxifeno, angiografía fluoresceínica, tomografía de coherencia óptica.

\begin{abstract}
Case report: We describe the case of a patient who presented with progressive and bilateral loss of vision. She had been treated with tamoxifen for 13 years. We performed fluorescein angiography and optical coherence tomography in order to study the macula.

Discussion: Loss of visual acuity related to tamoxifen maculopathy may be caused either by retinal nerve fibre atrophy or macular oedema. Macular findings obtained by fluorescein angiography and optical coherence tomography are complementary (Arch Soc Esp Oftalmol 2008; 83: 615-618).
\end{abstract}

Key words: Tamoxifen maculopathy, fluorescein angiography, optical coherence tomography.

\section{INTRODUCCIÓN}

El tamoxifeno es un fármaco con efecto antiestrogénico usado frecuentemente como tratamiento coadyuvante en el cáncer de mama. Se han descrito alteraciones oculares relacionadas con dicho fármaco a nivel de la córnea, retina y nervio óptico, aún utilizando dosis bajas del fármaco (1). En general, se estima que la prevalencia de toxicidad ocular causada por el tamoxifeno es baja (2). Los pacientes que muestran pérdida de visión en relación con el tamoxifeno, suelen presentar depósito de cristales blanco-amarillentos en la capa de fibras nerviosas e internas de la retina (3). En estos casos, es útil

\footnotetext{
Recibido: 24/9/07. Aceptado: 29/8/08.

Departamento de Oftalmología. Hospital de Sant Joan. Universitat i Virgili. Reus. España.

1 Licenciado en Medicina.

2 Doctor en Medicina.

Correspondencia:

M. Baget Bernaldiz

C/. Selva del Camp, 11

43392 Castellvell del Camp (Tarragona)

España

E-mail: mbaget@gmail.com
} 
el estudio mediante la angiografía fluoresceínica (AGF) ya que es frecuente observar la presencia de edema macular. Además, recientemente, se ha observado que el estudio complementario de la mácula en estos pacientes mediante la tomografía de coherencia óptica (OCT), puede ofrecer detalles anatómicos que no eran puestos de manifiesto con la $\operatorname{AGF}(4,5)$. El tratamiento de la maculopatía por tamoxifeno consiste en la discontinuación del fármaco, pudiendo observar mejoría de la visión en algunos enfermos.

\section{CASO CLÍNICO}

Se presenta el caso de una mujer de 53 años, que fue diagnosticada de cáncer de mama en junio del año 1992. La paciente fue sometida a mastectomía radical y se le prescribió tamoxifeno a razón de 20

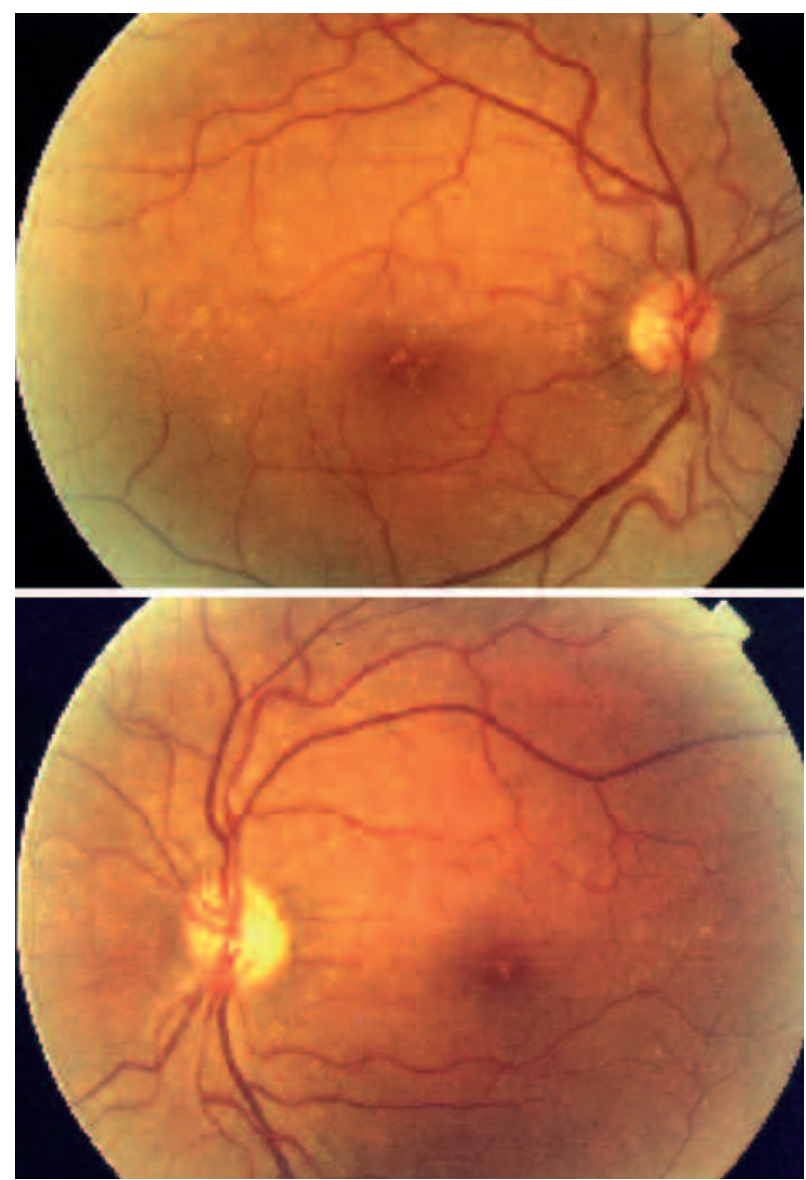

Fig. 1: Retinografía bilateral. Múltiples puntos blancoamarillentos dispersos en ambos polos posteriores. $\mathrm{mg} / \mathrm{d}$ que la paciente tomó regularmente durante 13 años (dosis acumulada $95 \mathrm{gr}$ ). En el último año de tomar dicho fármaco, la paciente presentó pérdida progresiva de la visión de forma bilateral. En el examen oftalmológico que se le practicó 6 meses después del inicio de los síntomas, se le objetivó una agudeza visual (AV) de 0,6 y 0,4 en el ojo derecho (OD) e izquierdo (OI), respectivamente. La presión intraocular así como el examen biomicroscópico resultaron ser normales. El fondo de ojo puso de manifiesto la presencia de múltiples puntos blancoamarillentos dispersos en ambos polos posteriores, con afectación de ambas fóveas (fig. 1). Se realizó estudio con AGF que puso de manifiesto la presencia de edema macular leve bilateral (fig. 2). El estudio mediante OCT, objetivó la existencia de un único quiste foveal con disrupción focal de la línea de fotorreceptores sin aumento del grosor macular en el OD y, disminución del grosor foveal en el OI (fig. 3).
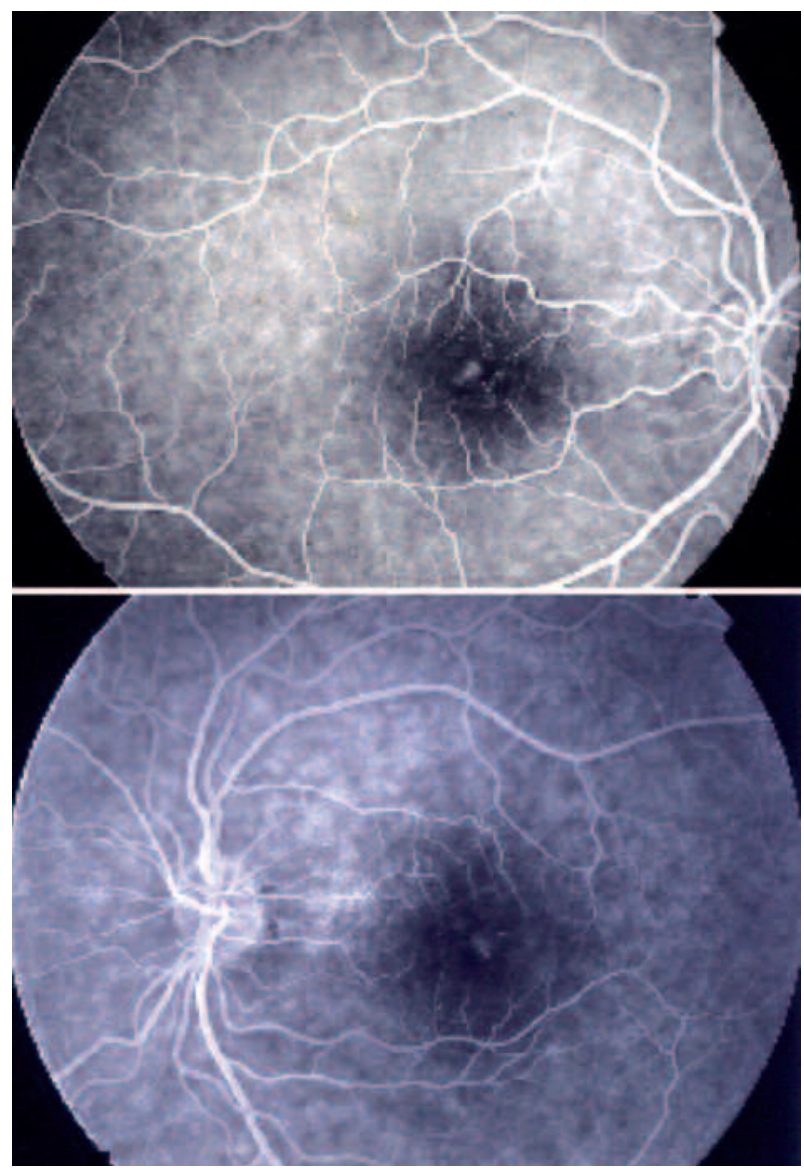

Fig. 2: Angiografía fluoresceína bilateral. Se aprecia escape leve de fluoresceína en ambas fóveas. 

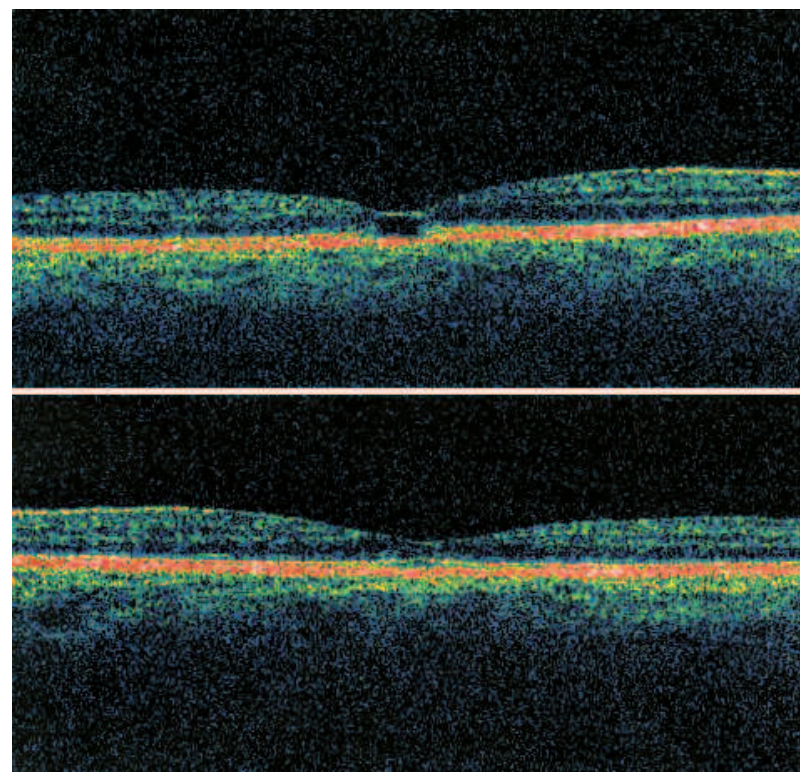

Fig. 3: OCT bilateral. Se observa un quiste foveal con disrupción focal de la línea de fotorreceptores en el $O D$ $y$ atrofia foveal en el OI.

El diagnóstico diferencial lo realizamos principalmente con las drusas dominantes y el fundus albipunctatus. La paciente no refería antecedentes de pérdida de visión prematura en ninguno de sus progenitores y no presentaba depósito significativo de cristales en el lado nasal del nervio óptico, zona típica de afectación en caso de drusas dominantes. Además, no refería nictalopía y la función escotópica del electroretinograma fue normal. La función fotópica del ojo izquierdo resultó estar levemente deprimida siendo el electroculograma normal en ambos ojos. La paciente fue diagnosticada de retinopatía por tamoxifeno y se recomendó la discontinuación del fármaco. A los 12 meses de la suspensión del fármaco, la AV mejoró de forma leve, únicamente en el OD llegando a ser de 0,.75. Desde entonces y a los 2 años de seguimiento, la AV se ha mantenido estable. El patrón de OCT se ha mantenido estacionario durante este tiempo.

\section{DISCUSIÓN}

El estudio de la maculopatía debido al tamoxifeno, clásicamente, se ha venido realizando mediante el examen de F.O. y la AGF. El caso que aquí presentamos, sometido únicamente al examen de F.O. y AGF, hubiera conllevado el diagnóstico de edema macular leve bilateral pero, al realizar el OCT, se pudo objetivar con mayor detalle la patología macular. Por lo tanto, al igual que han observado Gualiano et al. (4) y Bourla et al. (5), no siempre existe una correspondencia entre una evidencia angiográfica de edema macular y la presencia de dicho edema por OCT. En consecuencia, creemos que el OCT de manera complementaria a la AGF, puede ofrecer detalles anatómicos de la mácula que justifiquen mejor los síntomas visuales y quizás ayudar a establecer un pronóstico visual para estos enfermos. En nuestra paciente, se observó mejoría visual a los 12 meses de suspender el fármaco en el ojo del quiste foveal (OD) no siendo así en el ojo con atrofia de la fóvea ya establecida (OI). Hace falta la publicación de más casos de maculopatía por tamoxifeno analizados por OCT para tipificar los diferentes patrones maculares posibles y su correspondiente pronóstico visual.

\section{BIBLIOGRAFÍA}

1. Pavlis NA, Petris C, Briassoulis E, Klouvas G, Psilas C, Repapis J, et al. Clear evidence that long-term, low-dose tamoxifen treatment can induce ocular toxicity. A prospective study of 63 patients. Cancer 1992; 69: 2961-2964.

2. Heier JS, Dragoo RA, Enzenauer RW, Waterhouse WJ. Screening for ocular toxicity in asymptomatic patients treated with tamoxifen. Am J Ophthalmol 1994; 117: 772-775.

3. Mittra R, Mieler W, Drug toxicity of the posterior segment. In: Ryan SJ. Retina. St. Louis: Mosby; 2001; II: 1790.

4. Gualino V, Cohen SY, Delyfer MN, Sahel JA, Gaudric A. Optical coherente tomography findings in tamoxifen retinopathy. Am J Ophthalmol 2005; 140: 757-758.

5. Bourla DH, Sarraf D, Schwartz SD. Peripheral retinopathy and maculopathy in high-dose tamoxifen theraphy. Am J Ophthalmol 2007; 144: 126-128. 